\title{
EDITORIAL
}

\section{5 years after Robert Koch's discovery of the tubercle bacillus: the new XDR-TB threat. Is "science" enough to tackle the epidemic?}

\author{
G.B. Migliori*, R. Loddenkemper\# ${ }^{\#}$ F. Blasi ${ }^{\mp}$ and M.C. Raviglione ${ }^{+}$
}

$\mathbf{M}$ arch 24, 2007 will mark the 125th anniversary of Robert Koch's discovery of the tuberculosis (TB) bacillus (figs 1 and 2). This anniversary comes after a year of major achievements for the international TB community.

Firstly, in 2006, the new Stop TB Strategy was launched by the World Health Organization (WHO), following intensive exploration and discussion with $\mathrm{TB}$ control programme managers in high burden countries and partner organisations, including technical agencies and donors [2]. The DOTS strategy (consisting of five key elements: government commitment, diagnosis through microscopy, standardised and supervised treatment, uninterrupted drug supply, and regular programme monitoring), which greatly contributed to the improvement of global TB control over the last $10 \mathrm{yrs}$ [3], was revisited to pursue DOTS expansion while making explicit six additional components that must be implemented to reach the 2015 Millennium Development Goals (MDGs) relevant to TB control. Thus, the new Stop TB Strategy consists of: 1) the pursuit of high-quality DOTS expansion and enhancement; 2) addressing TB/HIV, multidrug-resistant (MDR)-TB and other challenges; 3) contribution to the strengthening of health systems; 4) engaging all care providers; 5 ) the empowerment of people and communities with $\mathrm{TB}$; and 6) enabling and promoting research. The MDGs relevant to $\mathrm{TB}$ aim at combating HIV, malaria and other diseases. Its target 8 consists of halting TB by 2015 and beginning to reverse the incidence of disease; indicator 23 addresses TB prevalence and mortality; and indicator 24 is the proportion of cases detected and cured under DOTS.

Secondly, the new Global Plan to Stop TB, 2006-2015 was launched in January 2006 [4]. The plan, underpinned by the Stop TB Strategy, describes strategies, financial requirements and existing gaps in reaching the MDGs throughout the world.

\footnotetext{
*WHO Collaborating Centre for Tuberculosis and Lung Diseases, Fondazione S. Maugeri, Care and Research Institute, Tradate, and, ${ }^{*}$ German Central Committee against Tuberculosis, Berlin, Germany. "Istituto Malattie Respiratorie, Università degli Studi di Milano, Padiglione Sacco, Ospedale Maggiore, IRCCS Fondazione Policlinico-Mangiagalli-Regina Elena, Milan, Italy. ${ }^{+}$Stop TB Dept, World Health Organization, Geneva, Switzerland.
}

STATEMENT OF INTEREST: None declared.

CORRESPONDENCE: G.B. Migliori, WHO Collaborating Centre for TB and Lung Diseases/TBNET Secretariat/Stop TB Italy, Fondazione S. Maugeri, Care and Research Institute, via Roncaccio 16, 21049, Tradate, Italy. Fax: 39 0331829402. E-mail: gbmigliori@fsm.it
It identifies a need for US\$56 billion over the decade, of which US $\$ 47$ billion are required for the implementation of countrywide programmes, including activities related to HIVassociated TB, the approach to MDR-TB (formerly referred to as "DOTS-Plus"), and the engagement of nonstate providers. The remaining US\$9 billion is needed for the research and development of new tools. Considering the significant prevalence of MDR and extensively drug-resistant (XDR) cases in several countries of the former Soviet Union, Europe will benefit from $20 \%$ of this budget, although it contributes with only $5 \%$ of the global burden of TB cases.

Thirdly, new International Standards of TB Care (ISTC) have been published and widely distributed, to guide both public and private healthcare providers to deliver quality DOTS services [5].

Finally, the new WHO guidelines on drug-resistant TB management have been published, stressing the importance of proper use of second-line drugs required to treat drugresistant and MDR-TB cases [6].

The year 2006 will also be remembered for the appearance of extensively drug-resistant TB (XDR-TB), a very serious form of TB against which our armamentarium has lost virtually all of its most powerful ammunitions [7]. The aim of this editorial is to describe XDR-TB, focusing on the mechanism determining its selection and spread, the implications and challenges that XDR-TB poses to TB control, and the possible approaches with which to tackle it.

\section{XDR-TB: DEFINITION}

The term XDR-TB was used for the first time in March 2006, in a report jointly published by the US Centers for Disease Control and Prevention (CDC) and WHO [7] to describe a disease caused by strains of Mycobacterium tuberculosis that were resistant not only to isoniazid and rifampicin (i.e. MDR$\mathrm{TB})$ but also to at least three of the six classes of second-line anti-TB drugs (aminoglycosides, polypeptides, fluoroquinolones, thioamides, cycloserine and para-aminosalycilic acid). As the definition is dependent on difficult-to-perform drug susceptibility testing (DST) and as some forms of drug resistance are less treatable then others, it was eventually modified at meeting of the WHO XDR-TB Task Force, on 1011 October 2006, in Geneva (Switzerland). Thus, XDR-TB is now defined as: "resistance to at least rifampicin and isoniazid 


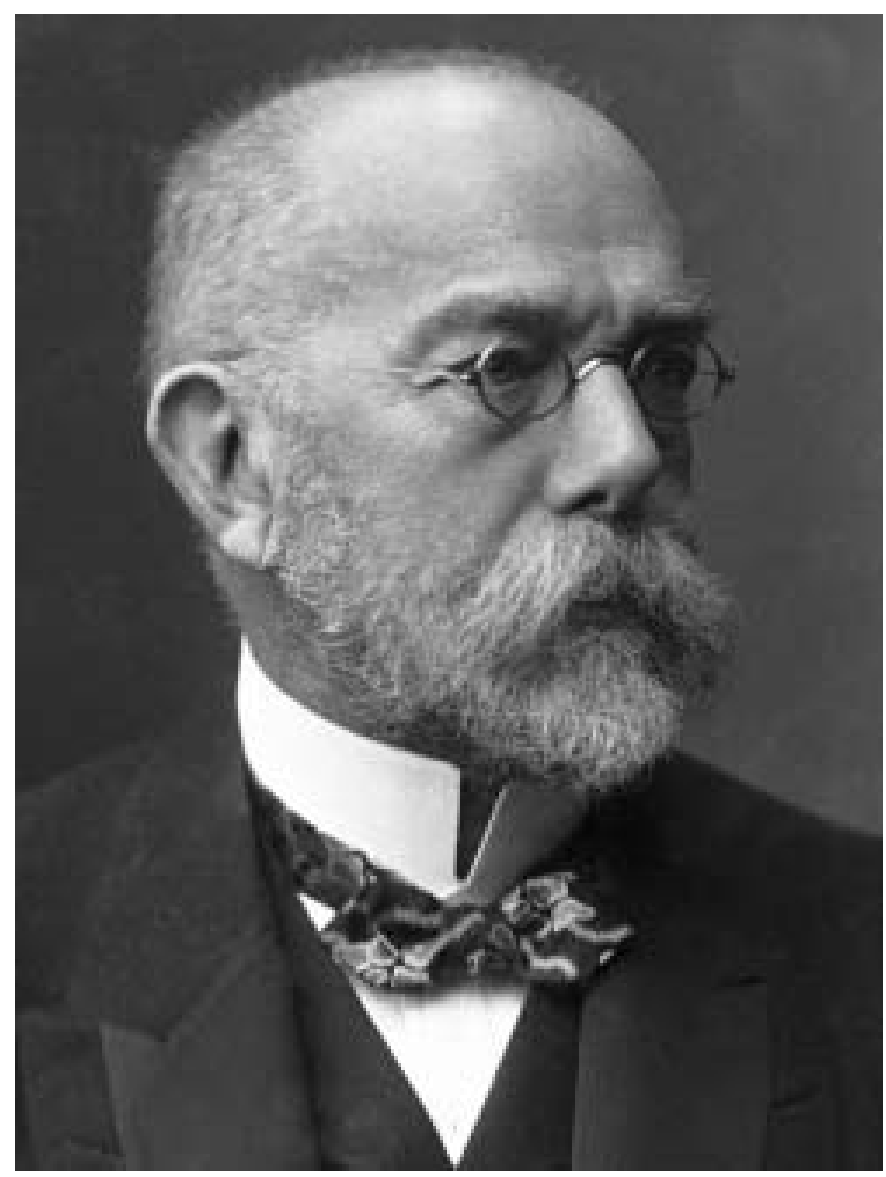

FIGURE 1. Robert Koch (1843-1910), who discovered Mycobacterium tuberculosis in 1882. Nobel Laureate Physiology or Medicine, 1905.

(which is the definition of MDR-TB), in addition to any fluoroquinolone, and to at least one of the three following injectable drugs used in anti-TB treatment: capreomycin, kanamycin and amikacin." $[8,9]$.

\section{XDR-TB EPIDEMIOLOGY AND SPREAD}

Due to its fairly recent definition, information about XDR-TB is still scattered and incomplete. The CDC study [7] reported that XDR-TB had been observed in all continents. Unpublished analysis of that data reveals that some 17 countries had at least one case of XDR-TB. More representative information from some countries has described the frequency of XDR-TB. For instance, in the USA, $4 \%$ of the MDR-TB strains isolated between 1993 and 2004 were XDR-TB. In Asia, data are available from South Korea [7], where 15\% of the MDR-TB strains isolated in 2004 were XDR, and also Iran [10], where 12 $(10.9 \%)$ of 113 MDR-TB strains isolated were XDR. In Hong Kong, nine out of the 75 MDR-TB strains (12\%) had extensive drug resistance, defined as simultaneous resistance to ethionamide, amikacin, ofloxacin and cycloserine [11]. In Europe, representative data are available from Latvia [7], where 19\% of the MDR strains isolated from 2000-2002 were XDR. In addition, XDR-TB was signalled from Russia (one XDR case resistant to ethionamide, kanamycin and ofloxacin, identified out of the 77 MDR strains tested [12]) and Norway, where an outbreak of 23 cases ( 15 of them being XDR) has been ongoing for $>10$ yrs [13].

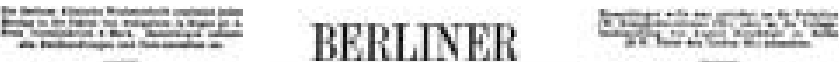 KLINISCHE WOCHENSCHRIFT.}

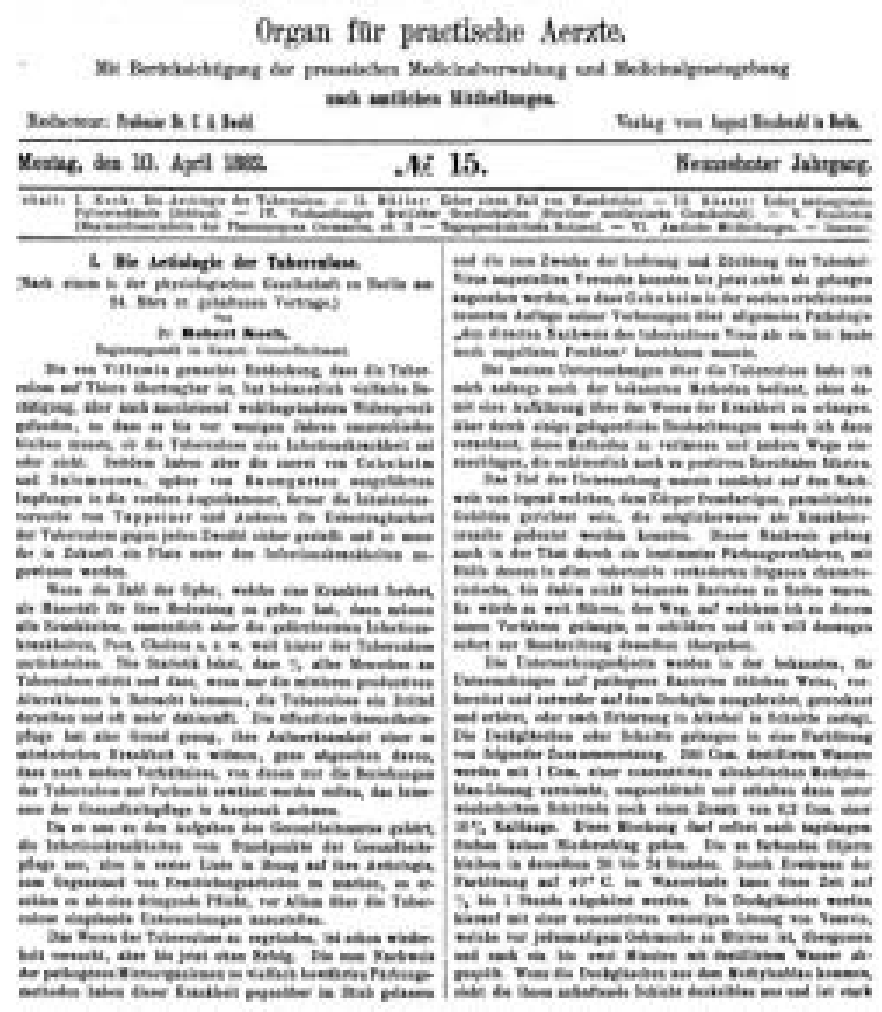

FIGURE 2. The title page of Robert Koch's original article published in Berliner Klinische Wochenschrift on April 10, 1882 [1]. This journal is no longer in existence. Provided courtesy of H.L. Rieder (Tuberculosis Division, International Union Against Tuberculosis and Lung Disease, Paris, France).

In Africa, data are currently only available from South Africa [14]. In the Msinga district, KwaZulu Natal (KZN), out of 1,539 TB cases diagnosed between January 2005 and March 2006, 542 were culture positive, 221 were MDR with 53 "possible" XDR cases. Out of the 53 XDR patients, 52 died (with a median survival time after sputum smear sample collection of only 16 days). In total 44 cases tested were infected with HIV: 15 died while receiving antiretroviral treatment and two of them were healthcare workers (HCW). Of the XDR patients, 26 (51\%) were new cases. Although $64 \%$ of XDR cases had been admitted to hospital before the diagnosis of $\mathrm{TB}$, a third of them $(36 \%)$ had no history of hospitalisation and may have acquired infection at community level. Spoligotyping results demonstrated that $85 \%$ of the 46 XDR strains tested belonged to the KZN family, and amongst the remaining $15 \%$, some belonged to the Beijing family of strains. The strains were all resistant to all first-line drugs and to two classes of second-line drugs: aminoglycosides (kanamycin and amikacin) and fluoroquinolones. The strains were susceptible to ethionamide and cycloserine; however, the validity of DST for these drugs is uncertain. Susceptibility to capreomycin and para-aminosalycilic acid was not tested, as these drugs were not, until recently, available in South Africa. 
The available information on the global spread of MDR and XDR-TB is summarised in figure 3; note that out of the 21 countries where at least one XDR-TB case was notified, 10 are in, or bordering, Europe.

In most of the world XDR-TB seems to have appeared among HIV-seronegative individuals, the epidemic of XDR-TB among people living with HIV/AIDS in South Africa represents a serious challenge. While the true mechanism responsible for such an event is still not entirely clear, a number of cases in KZN resulted from in-hospital infection. It is likely, however, that other factors linked to poor control practices (for example, lack of measures to ensure adherence to treatment) played a major role in originating XDR-TB cases. The appearance of cases among HIV-infected individuals is, in any case, very concerning, as rapid progression of infection towards disease and the high potential of spread among immunosuppressed individuals greatly accelerate the consequences of bad control practices. In Europe and elsewhere, where the majority of cases are not among persons living with HIV/AIDS, the event may present with a more chronic evolution and may not be as noticeable as in South Africa [15].

\section{IMPLICATIONS AND CHALLENGES FOR TB CONTROL}

The major implications for TB control were discussed at an Expert Consultation meeting in Johannesburg (South Africa) in September 2006, jointly organised by the South Africa Medical
Research Council, CDC and the WHO. The main priority interventions discussed were threefold, namely: 1) the strengthening of TB control (through sound implementation of the Stop TB Strategy and systematic application of the ISTC in both the public and private sector with focus on laboratory capacities and infection control); 2) improvement of secondline drug management based on the new WHO guidelines [4]; and 3) promotion of research and development of new diagnostics, vaccines and drugs [2].

\section{PROPOSED SOLUTIONS FOR XDR-TB PREVENTION AND CONTROL}

The WHO Global Task Force on XDR-TB that met in Geneva on 9-10 October 2006 developed seven main recommendations to prevent and control XDR, as follows.

1) Prevention of $X D R-T B$ through basic strengthening of $T B$ and HIV control. The new Stop TB Strategy and the Global Plan to Stop TB are the essential reference documents to guide these priority interventions.

2) Improvement of management of XDR-TB suspects. These interventions will be based on accelerating the access to laboratory facilities, with DST including a rapid test for rifampicin resistance, and improving detection of cases suspected of harbouring MDR strains both in high and low HIV prevalence settings.

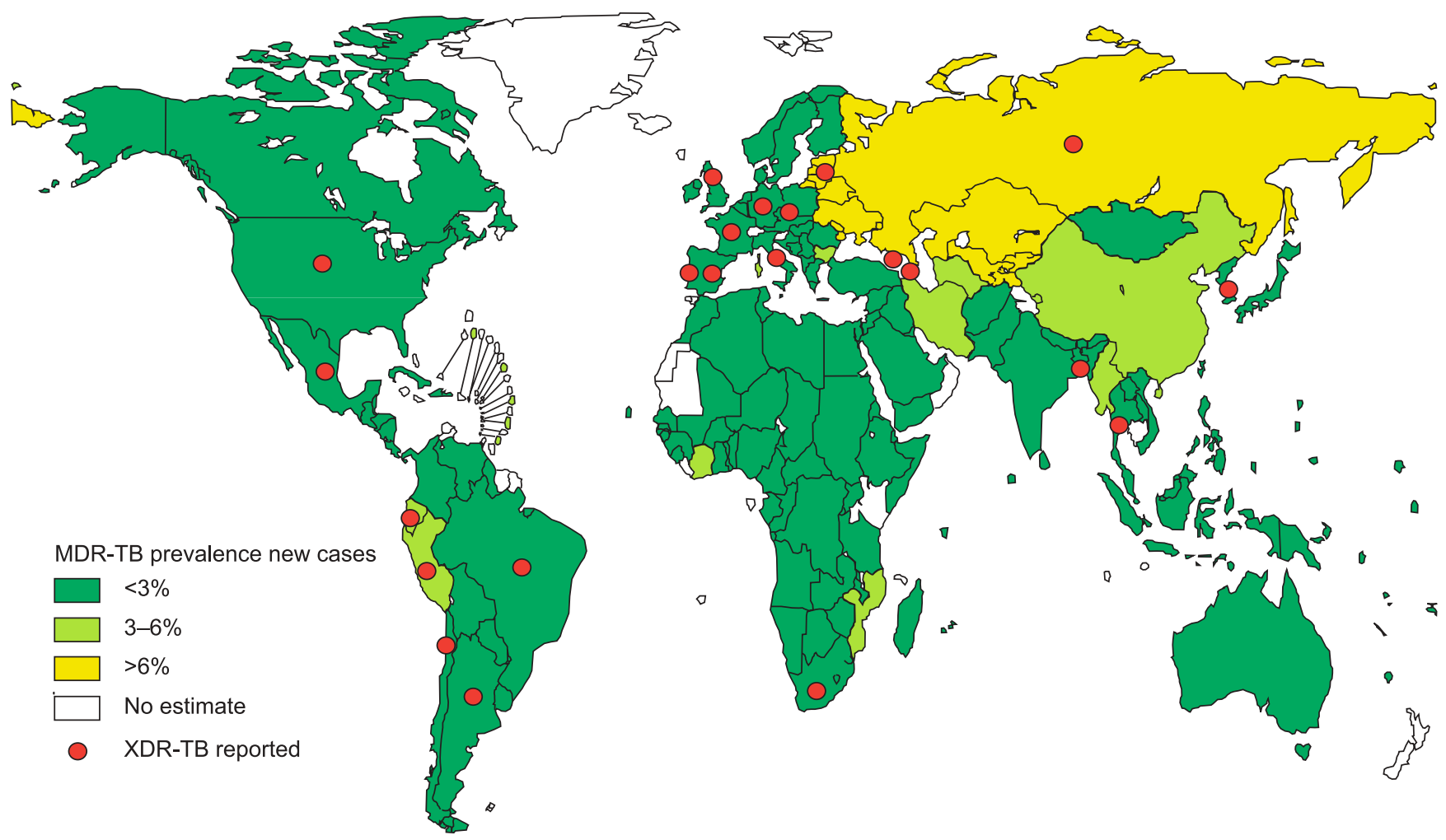

FIGURE 3. The global prevalence of multidrug-resistant tuberculosis (MDR-TB) among new cases and the countries in which at least one case of extensively drugresistant TB (XDR-TB) was described. MDR: cases resistant to at least rifampicin and isoniazid. XDR: resistance to at least rifampicin and isoniazid, in addition to any fluoroquinolone, and to at least one of the three following injectable drugs used in anti-TB treatment: capreomycin, kanamycin and amikacin. The following countries had at least one case of XDR-TB described: Argentina, Armenia, Bangaldesh, Brazil, Chile, Czech Republic, Ecuador, France (one case imported), Georgia, Germany, Italy, Republic of Korea, Latvia, Mexico, Peru, Portugal, Russian Federation, South Africa, Spain, Thailand, the UK and the USA. Figure reproduced from [4] with permission from the Stop TB Department, World Health Organization, Geneva, Switzerland. 
3) Strengthened management of XDR-TB and treatment design in HIV-negative and -positive individuals. This intervention will be based on the adequate application of the new WHO guidelines for programmatic management of drug-resistant TB using second-line drugs properly and through a patientcentred approach to ensure adequate support and supervision.

4) Standardisation of the definition of XDR-TB. The global adoption of the new definition (described previously) will improve the comparability of data obtained through ongoing surveillance in low TB incidence and ad hoc surveys in high TB incidence countries (see point 6).

5) An increase in HCW infection control and protection. This intervention, aimed at reducing the ongoing transmission of MDR-TB especially among HIV-positive individuals in congregate care settings, will be focused mainly (but not exclusively) on high HIV prevalence settings.

6) Implementation of immediate XDR-TB surveillance activities. Supranational and National Reference Laboratories will need to initiate rapid surveys in early 2007 to allow a complete assessment of the XDR prevalence worldwide.

7) Initiation of advocacy, communication and social mobilisation activities. There is an urgent need to inform and raise awareness about TB and XDR-TB.

\section{ROLE OF SCIENTIFIC SOCIETIES AND THE EUROPEAN RESPIRATORY SOCIETY IN THE PREVENTION AND CONTROL OF XDR IN EUROPE}

Most of the countries with a high drug resistance burden are in Eastern Europe. According to a recent study [16] $>63,000$ MDR-TB cases have been estimated to occur in the countries of the former Soviet Union. Thus, European scientific societies may play a substantial role in disseminating the relevant guidelines among their members and promoting their correct application as part of good clinical practices. The European Respiratory Society (ERS) has been particularly active in supporting TB and XDR-TB control in the last couple of months, through the following actions.

1) The ISTC were endorsed and an editorial targeting ERS members was published [17].

2) The ERS joined with the European Stop TB Partnership, which is composed of donors, technical agencies and other stakeholders, committed to fight against TB (MDR-TB and XDR-TB) in Europe. In this capacity the ERS will be represented at a Ministerial Conference (promoted by the WHO and Stop TB Partnership) in 22 October 2007 in Berlin (Germany), where ministers of health, foreign affairs, finance and other sectors of both Eastern and Western European countries will convene to discuss programmatic and financial issues related to TB control in those European countries facing high rates of TB and MDR-TB.

3) The ERS is supporting, within Assembly 10 (Respiratory Infections), the activities of the TB study group (Group 10.2: Tuberculosis) presently committed to developing human resources on TB control and promoting quality operational research in all European countries. Group 10.2 recently proposed to the Executive Committee of the ERS, a research project on the surveillance and control of XDR-TB in Europe.
Furthermore, within Assembly 10 and Group 10.2 a new initiative was developed. A network built to organise clinical trials on TB has recently been established in Borstel, Germany (9-10 November 2006), called TuBerculosis Network in Europe Trialsgroup (TBNET). Its new steering committee identified five priority studies to be supported, including: 1) evaluation of new interferon- $\gamma$ tests and new markers of TB infection and disease; 2 ) evaluation of the impact of national/ERS guidelines in Europe; 3) control of MDR/XDR-TB; 4) rapid diagnosis of smear-negative TB cases; and 5) a prospective study aimed at re-evaluating the breakdown rate (progression from infection to diseases) in truly infected individuals.

\section{CONCLUSIONS}

The evidence available shows that extensively drug-resistant tuberculosis is present in Europe, mainly as a result of poor clinical and control practices. Preliminary anecdotal evidence suggests that some extensively drug-resistant strains in Europe (from Italy, for example) may, in fact, be "XXDR" or "extremely drug-resistant", i.e. resistant to all first- and second-line drugs available. Basic science will need to provide a key contribution in developing new diagnostics, new drugs and, ultimately, a vaccine with which to fight tuberculosis. Unfortunately, several more years will be necessary to develop them. Even when new drugs are available, without a sound public health approach to using them, they may be rapidly lost. This has already happened to some of the key old drugs, "burnt" as a result of clinical and public health malpractices in many settings. Science is crucial but alone it is not sufficient to control the extensively drug-resistant tuberculosis epidemic. Exactly 125 years after Robert Koch's discovery of Mycobacterium tuberculosis (the milestone of modern control of TB) the "white plague" is still affecting mankind. While waiting for the much desired new diagnostic, treatment and prevention tools, public health is asked to provide a prompt response and curb the extensively drug-resistant epidemic using the available tools because affected people are dying today. The respiratory medicine specialists' contribution is crucial to win this battle.

\section{ACKNOWLEDGEMENTS}

The authors wish to thank D. Cirillo (S. Raffaele Institute, Milan, Italy), A. Matteelli (University of Brescia, Brescia, Italy) and V. Leimane (WHO collaborating Centre for Research and Training in Management of MDR-TB, State Agency of Tuberculosis and Lung Diseases, Riga, Latvia) for their useful comments. The authors also thank the staff (R. Centis and L. D'Ambrosio) of the WHO Collaborating Centre for TB and Lung Diseases (Fondazione S. Maugeri, Tradate, Italy) for their editorial support in the preparation of the manuscript.

\section{REFERENCES}

1 Koch R. Die Aetiologie der Tuberculose [The aetiology of Tuberculosis]. Berliner Klinische Wochenschrift 1882.

2 Raviglione MC, Uplekar M. WHO's new Stop TB Strategy. Lancet 2006; 367: 952-955.

3 Sharma SK, Liu JJ. Progress of DOTS in global tuberculosis control. Lancet 2006; 367: 950-952.

4 Stop TB Partnership and World Heath Organization. The Global Plan to Stop TB 2006-2015. Geneva, World Health Organization, 2006. 
5 Hopewell PC, Pai M, Maher D, Uplekar M, Raviglione MC. International Standards for Tuberculosis Care. Lancet Infect Dis 2006; 6: 710-725.

6 World Health Organization. Guidelines for the programmatic management of drug-resistant Tuberculosis. WHO/ HTM/TB/2006.361. Geneva, World Health Organization, 2006.

7 Centers for Disease Control and Prevention. Emergence of Mycobacterium tuberculosis with extensive resistance to second-line drugs-worldwide. MMWR Morb Mortal Wkly Rep 2006; 55: 301-305.

8 World Health Organization. Extensively drug-resistant tuberculosis (XDR.TB): recommendations for prevention and control. Weekly Epidemiol Record 2006; 81: 430-432.

9 The tuberculosis X factor. Lancet Infect Dis 2006; 6: 679.

10 Masjedi MR, Farnia P, Sorooch S, et al. Extensively drugresistant tuberculosis: 2 years of surveillance in Iran. Clin Infect Dis 2006; 43: 841-847.

11 Toungoussova OS, Mariandyshev AO, Bjune G, Caugant DA, Sandven P. Resistance of multidrug-resistant strains of Mycobacterium tuberculosis from the Archangel oblast, Russia, to second-line anti-tuberculosis drugs. Eur J Clin Microbiol Infect Dis 2005; 24: 202-206.

12 Kam KM, Yip CW. Surveillance of Mycobacterium tuberculosis susceptibility to second-line drugs in Hong Kong, 1995-2002, after the implementation of DOTS-plus. Int J Tuberc Lung Dis 2004; 8: 760-766.

13 Dahle UR. Extensively drug resistant tuberculosis: beware patients lost to follow-up. BMJ 2006; 333: 705.

14 Gandhi NR, Moll A, Sturm AW, et al. Extensively drugresistant tuberculosis as a cause of death in patients coinfected with tuberculosis and HIV in a rural area of South Africa. Lancet 2006; 368: 1575-1580.

15 Raviglione MC. XDR-TB: entering the post-antibiotic era? Int J Tuberc Lung Dis 2006; 10: 1185-1187.

16 Zignol M, Hosseini MS, Wright A, et al. Global incidence of multidrug-resistant tuberculosis. J Infect Dis 2006; 194: 479-485.

17 Migliori GB, Hopewell PC, Blasi F, Spanevello A, Raviglione MC. Improving the TB case management: the International Standards for Tuberculosis Care. Eur Respir J 2006; 28: 1-4. 